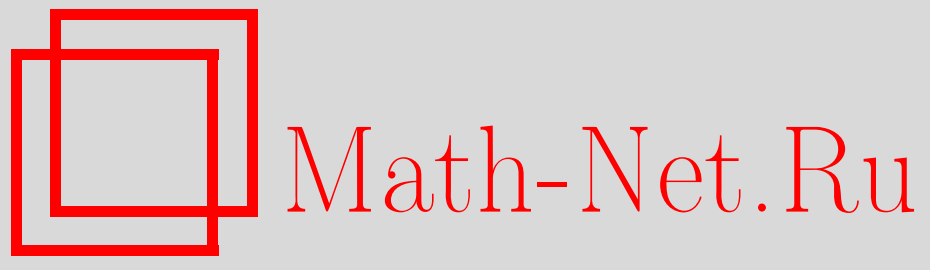

А. А. Гущин, М. А. Урусов, Процессы, вкладывающиеся в геометрическое броуновское движение, Теория вероятн. и ее примен., 2015, том 60, выпуск 2, 248-271

DOI: https://doi.org/10.4213/tvp4618

Использование Общероссийского математического портала Math-Net.Ru подразумевает, что вы прочитали и согласны с пользовательским соглашением http: //www . mathnet.ru/rus/agreement

Параметры загрузки:

IP : 54.162 .127 .20

26 апреля 2023 г., 18:14:05

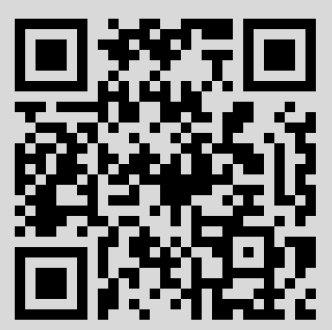




\section{ПРОЦЕССЫ, ВКЛАДЫВАЮЩИЕСЯ В ГЕОМЕТРИЧЕСКОЕ БРОУНОВСКОЕ ДВИЖЕНИЕ ${ }^{1)}$}

Основной результат является аналогом теоремы Монро (1978) в случае геометрического броуновского движения: случайный процесс эквивалентен замене времени в геометрическом броуновском движении тогда и только тогда, когда он есть неотрицательный супермартингал. Мы также указываем на связь нашего основного результата с работой Монро (1972). Эта связь основана на понятии минимального момента остановки и его характеризации в работах Монро (1972) и Кокса и Хобсона (2006) в случае броуновского движения. В заключение мы предлагаем достаточное условие для минимальности для процессов, отличных от броуновского движения, дополняя обсуждение в указанных работах.

Ключевые слова и фразы: геометрическое броуновское движение, вложение Скорохода, теорема Монро.

1. Введение и основной результат. В своей основополагающей работе [29] Монро доказал, что непрерывный справа и имеющий пределы слева процесс эквивалентен конечной замене времени в броуновском движении тогда и только тогда, когда он является семимартингалом. В статье процессы называются эквивалентными, если их распределения совпадают. Мы доказываем аналогичный результат для геометрического броуновского движения.

Теорема 1.1. (i) Пусть $X=\left(X_{s}\right)_{s \geqslant 0}$ - неотрицательньий супермартингал с $\mathbf{E} X_{0} \leqslant 1$. Тогда существуют такие вероятностное пространство с фильтрациеи $\left(\Omega, \mathscr{F}_{t},\left(\mathscr{F}_{t}\right)_{t \geqslant 0}, \mathbf{P}\right),\left(\mathscr{F}_{t}, \mathbf{P}\right)$-броуновское движение $W=\left(W_{t}\right)$ и $\left(\mathscr{F}_{t}\right)$-замена времени $\left(T_{s}\right)$ со значениями в $[0, \infty]$,

*Математический институт им. В.А. Стеклова РАН; Национальный исследовательский университет «Высшая школа экономики», Москва, Россия; е-таil: gushchin@mi.ras.ru

** Математический институт им. В. А. Стеклова РАН, Москва, Россия; Университет Дуйсбург-Эссен, Эссен, Германия; e-mail: mikhail.urusov@uni-due.de

1) Исследование А.А.Гущина в разд. 1-3 поддержано Международной лабораторией количественных финансов НИУ ВШЭ (грант Правительства РФ, дог. 14.А12.31.0007) и грантом РФФИ 14-01-00739. Исследование А. А.Гущина и М. А. Урусова в разд. 4 выполнено за счет гранта Российского научного фонда (проект № 14-21-00162) в Математическом институте им. В. А. Стеклова Российской академии наук. 
что процессьи $\left(X_{s}\right)_{s \geqslant 0}$ u $\left(Z_{T_{s}}\right)_{s \geqslant 0}$ имеют одинаковые распределения, где $Z_{t}=e^{W_{t}-t / 2}, t \geqslant 0$.

(ii) Обратно, для любой $\left(\mathscr{F}_{t}\right)$-замень времени $\left(T_{s}\right)$ со значениями в $[0, \infty]$ прочесс $\left(Z_{T_{s}}\right)$ есть неотрицательньй $\left(\mathscr{F}_{T_{s}}, \mathbf{P}\right)$-супермартингал $c$ $\mathbf{E} Z_{T_{0}} \leqslant 1$.

Часть (ii) немедленно следует из теоремы Дуба о преобразовании свободного выбора для неотрицательных супермартингалов, примененной к $\left(Z_{t}\right)$, так что в доказательстве нуждается только часть (i). Заметим также, что моменты остановки $T_{s}$ в теореме 1.1 с необходимостью являются минимальньли для $X$ (ср. с разд. 4, в частности, с замечанием 4.7).

Мы по умолчанию работаем с непрерывными справа и имеющими пределы слева процессами. В частности, «супермартингал» означает «непрерывный справа и имеющий пределы слева супермартингал». Напомним, что замена времени - это такое семейство $\left(T_{s}\right)_{s \geqslant 0}$ моментов остановки, что отображения $s \mapsto T_{s}$ п.н. не убывают и непрерывны справа. В отличие от постановки задачи в [29] здесь не требуется, чтобы моменты остановки $T_{s}$ принимали конечные значения. В нашей постановке это естественно, так как неотрицательный мартингал $\left(Z_{t}\right)$ имеет предел $Z_{\infty} \equiv 0$ и замкнут справа как супермартингал посредством этого предела. ${ }^{2)}$

С одной стороны, часто полезно знать, можно ли рассматривать случайный процесс, как получающийся заменой времени из процесса простой структуры. В финансах подход к моделированию, основанный на замене времени, фактически начался с теоремы Монро, см. [2]. В настоящее время этот подход к моделированию очень популярен в финансовой математике, см. [6] и ссылки в ней. С другой стороны, теорема Монро одна из производных задачи вложения Скорохода. Первоначально эта задача была сформулирована и решена в [37] (английский перевод — [36]), что привело к появлению большого количества литературы. Всесторонний обзор состояния задачи вложения Скорохода к 2004 году приведен в [30], где, в частности, описаны более двадцати различных подходов к решению задачи и соотношения между ними, различные постановки и обобщения, а также некоторые производные задачи. Мотивация Скорохода к решению задачи состояла в доказательстве предельных теорем (например, для того чтобы получить закон повторного логарифма для случайных блужданий из этого закона для броуновского движения), но в последние годы появились и другие применения. Методология, основанная на вложении Скорохода и потраекторных неравенствах, оказалась

2) Немедленным следствием теоремы 1.1 является утверждение, получаемое из теоремы 1.1 заменой слов «неотрицательный» на «строго положительный» и «со значениями в $[0, \infty] \gg$ на «с конечными значениями». 
важной для нахождения независимых от модели границ цен опционов и робастных хеджирующих стратегий. ${ }^{3)}$ Это привело к дальнейшим исследованиям в данном направлении, которые продолжаются до сих пор, см., например, [1], [8], [10], [14], [15]-[18], [22]. Больше деталей и много дальнейших ссылок можно найти в недавних обзорах [23] и [31] по задаче вложения Скорохода и ее применениям к робастному ценообразованию и хеджированию.

Несмотря на общность теоремы Монро, мы не можем вывести теорему 1.1 как ее следствие (причина описана в разд. 3) и поэтому доказываем теорему 1.1 независимо. Доказательство следует работе Монро [29], хотя некоторые технические детали проработаны иначе, что связано с естественными различиями между постановками. На первом шаге мы рассматриваем задачу вложения Скорохода для геометрического броуновского движения, т.е. вложение одной случайной величины в геометрическое броуновское движение. Различные решения этой задачи (в действительности, вложение в броуновское движение со сносом) были предложены в [21], [19], [34], [3]-[5]. В разд. 2.1 предлагается альтернативный вариант конструкции, который, на наш взгляд, интересен сам по себе. Также эта конструкция будет полезна в разд. 2.2. В литературе встречались явные вложения в однородные во времени диффузии, см. [33], [12], [4], разд. 9 из [30], разд. 4.3 из [23] и содержащиеся в них ссылки. Однако, насколько нам известно, конструкция, описанная в разд. 2.1, не появлялась в работах по этой тематике, хотя идеи, стоящие за ней, безусловно, встречаются в литературе. В частности, наша конструкция может рассматриваться как переработка оригинальной конструкции Скорохода (см. [37] или разд. 3.12 в [23]) или конструкции Монро в [29] для случая геометрического броуновского движения. На втором шаге мы вкладываем супермартингал с дискретным временем в геометрическое броуновское движение. Как правило, если известно, как вложить одну случайную величину, то задача вложить процесс с дискретным временем сложности не вызывает. Однако в нашей ситуации этот шаг, на удивление, связан с техническими сложностями. Их причина состоит в том, что замена времени может принимать бесконечное значение, см. подробности в разд. 2.2. На третьем шаге мы обосновываем переход к пределу с непрерывным временем. Эта часть ближе к соответствующей части доказательства теоремы Монро, и мы, на самом деле, в определенный момент просто отсылаем читателя к [29] (см. разд. 2.3). В разд. 3 обсуждаются некоторые вопросы, связанные с минимальными моментами остановки для броуновского движения, поня-

3) Отметим, что робастные методы хеджирования могут часто превосходить классическое (определяемое моделью) хеджирование в случае неопределенности модели и/или рынков с трением, см. [32]. 
тием, изучаемом в другой работе Монро [28] и встречающемся во многих последующих работах о задаче вложения Скорохода и порожденных ею задачах. В частности, мы объясняем, почему теорема 1.1 не является следствием результатов работ [28] и [29], и приводим в теореме 3.4 эквивалентную формулировку теоремы 1.1, которая дополняет обсуждение в [28]. Для броуновского движения минимальность охарактеризована в [28] и, для более общего случая, в [13]. В разд. 4 изучаются минимальные моменты остановки для других процессов. А именно, в теореме 4.1 мы даем достаточное условие минимальности, которое является новым и дополняет обсуждения минимальных моментов остановки для процессов, отличных от броуновского движения, в [30, разд. 8], [23, разд. 3.4] и [31, разд. 2.2]. Мы увидим, что теорема 4.1 применима во многих ситуациях.

Закончим введение обсуждением вложения в процесс $\bar{Z}_{t}^{a, b}=e^{a W_{t}+b t}$, $t \geqslant 0$, где $a \neq 0, b \in \mathbf{R}$. Пусть сначала $b \neq 0$, тогда (возможно, бесконечный) предел $\bar{Z}_{\infty}^{a, b}:=\lim _{t \rightarrow \infty} \bar{Z}_{t}^{a, b}$ определен, т.е. естественно рассматривать замены времени со значениями в $[0, \infty]$. Тогда из теоремы 1.1 следует, что непрерывный справа и имеющий пределы слева процесс $\bar{X}$ эквивалентен замене времени со значениями в $[0, \infty]$ в процессе $\bar{Z}^{a, b}$ тогда и только тогда, когда $\bar{X}^{\lambda}$ является неотрицательным супермартингалом с $\mathbf{E} \bar{X}_{0}^{\lambda} \leqslant 1$, где $\lambda=-2 b / a^{2}$. Заметим, что $\bar{X}$ может принимать значение $+\infty$ в случае $b>0$. Пусть теперь $b=0$. Поскольку $\lim _{t \rightarrow \infty} \bar{Z}_{t}^{a, 0}$ не существует, естественно рассматривать только конечные замены времени. Тогда по теореме Монро непрерывный справа и имеющий пределы слева процесс $\bar{X}$ эквивалентен конечной замене времени в $\bar{Z}^{a, 0}$ тогда и только тогда, когда это строго положительный семимартингал.

\section{2. Доказательство теоремы 1.1.}

2.1. Вложение одной случайной величины. Будем использовать обозначения $\mu_{W}$ для винеровской меры на $\left(\mathbf{C}\left(\mathbf{R}_{+}\right), \mathscr{B}\left(\mathbf{C}\left(\mathbf{R}_{+}\right)\right)\right)$и $\mu_{L}$ для меры Лебега на $([0,1], \mathscr{B}([0,1]))$. Для некоторых случайных величин $\xi$ и $\eta$ пишем $\xi \sim \eta$ для обозначения того, что $\xi$ и $\eta$ имеют одинаковое распределение.

Лемма 2.1. Пусть $\xi-$ неотрииательная случайная величина $c$ $\mathbf{E} \xi \leqslant 1$. Рассмотрим вероятностное пространство с фильтраиией $\left(\Omega, \mathscr{F},\left(\mathscr{F}_{t}\right)_{t \geqslant 0}, \mathbf{P}\right)$, гдe

$$
\Omega=\mathbf{C}\left(\mathbf{R}_{+}\right) \times[0,1], \quad \mathscr{F}=\mathscr{B}\left(\mathbf{C}\left(\mathbf{R}_{+}\right)\right) \otimes \mathscr{B}([0,1]), \quad \mathbf{P}=\mu_{W} \times \mu_{L}
$$

$u \mathscr{F}_{t}=\bigcap_{\varepsilon>0} \sigma\left(R, B_{s} ; s \in[0, t+\varepsilon]\right)$, а случайная величина $R$ и прочесс $B=$ $\left(B_{t}\right)$ на $\Omega$ определень следующим образом: если $\omega=(x, r)$, то $R(\omega):=r$, $B_{t}(\omega):=x(t) . B$ частности, $R$ является $\mathscr{F}_{0}$-измеримой и равномерно распределенной на $[0,1]$, а $B-\left(\mathscr{F}_{t}, \mathbf{P}\right)$-броуновское движение. Тогда 
существует такой $[0, \infty]$-значньии $\left(\mathscr{F}_{t}\right)$-момент остановки $\tau$, ито $\xi \sim$ $Y_{\tau}$, где $Y_{t}=e^{B_{t}-t / 2}, t \geqslant 0$.

Отметим здесь, что обратное, очевидно, также выполнено, т.е. случайная величина может быть вложена в геометрическое броуновское движение тогда и только тогда, когда она неотрицательна и ее математическое ожидание меньше или равно единице.

Д о к а з а т ель с т в о. Пусть $F-$ функция распределения $\xi$. Определим квантильную функцию $F^{-1}:[0,1] \rightarrow[0,+\infty]$ как непрерывную справа обратную функцию к $F$, т.е. $F^{-1}(r)=\inf \left\{x \in \mathbf{R}_{+}: F(x)>r\right\}$; здесь и далее inf $\varnothing=+\infty$. Хорошо известно, что $F^{-1}(R)$ имеет такое же распределение, как и $\xi$. Поэтому далее без потери общности считаем, что $\xi=F^{-1}(R)$.

Положим $h(r)=\int_{0}^{r} F^{-1}(s) d s, g(r)=r-h(r), r \in[0,1]$. Тогда $h-$ неубывающая выпуклая функция на $[0,1], h(0)=0, h(1)=\mathbf{E} \xi \leqslant 1$. Если $h(1)<1$, то уравнение $g(x)=c$ для $0 \leqslant c<g(1)$ имеет ровно одно решение, скажем, $\theta=\theta(c) \in[0,1]$. Для такого $\theta$ положим $U(\theta)=F^{-1}(\theta)$, $V(\theta)=+\infty$. Если $g(1) \leqslant c<g^{*}:=\max _{r \in[0,1]} g(r)$, то это же уравнение имеет два решения $\theta_{1}<\theta_{2}$ в $[0,1]$. Для таких $\theta_{1}$ и $\theta_{2}$ положим $U\left(\theta_{1}\right)=U\left(\theta_{2}\right)=F^{-1}\left(\theta_{1}\right), V\left(\theta_{1}\right)=V\left(\theta_{2}\right)=F^{-1}\left(\theta_{2}\right)$. Для тех значений $\theta \in[0,1]$, для которых $g(\theta)=g^{*}$, положим $U(\theta)=V(\theta)=1$. Таким образом, мы определили функции $U:[0,1] \rightarrow[0,1]$ и $V:[0,1] \rightarrow[1,+\infty]$. Наконец, введем случайные величины $\eta:=g(R), \alpha:=U(R)$ и $\beta:=V(R)$. Отметим, что $\alpha$ и $\beta$ на самом деле являются функциями от $\eta$. На рис. 1 мы объясняем структуру случайных величин $\xi, \alpha$ и $\beta$ с помощью графиков функций $h$ и $g$.
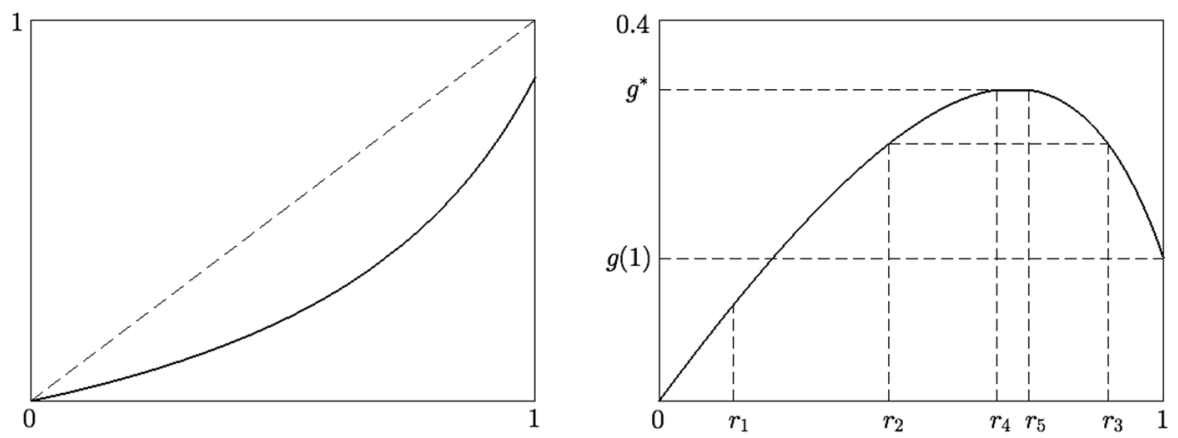

Рис. 1. На рисунке слева сплошная линия - график функции $r \mapsto h(r)$, штриховая линия - график тождественной функции $r \mapsto r$. Связь со структурой случайной величины $\xi$ объясняется формулой $\xi=F^{-1}(R)=h_{+}^{\prime}(R)$, где $h_{+}^{\prime}-$ правая производная выпуклой функции $h$. На рисунке справа сплошная линия - график функции $r \mapsto g(r)$. Структуру случайных величин $\alpha$ и $\beta$ можно объяснить следующим образом: если $R=r_{1}$, то $\alpha=F^{-1}\left(r_{1}\right)=\xi$ и $\beta=+\infty$; если $R \in\left\{r_{2}, r_{3}\right\}$, то $\alpha=F^{-1}\left(r_{2}\right)$ и $\beta=F^{-1}\left(r_{3}\right)$; если $R \in\left[r_{4}, r_{5}\right]$, то $\alpha=\beta=1$. 
Ключевым моментом нашей конструкции является следующая характеризация условного распределения $\xi$ относительно $\eta$ :

(а) п.н. при событии $\{\eta<g(1)\}$ распределение сосредоточено на одноточечном множестве $\{\xi\}$ (обратите внимание, что $\xi-$ функция от $\eta$ при этом событии, потому что $R$ и $\eta$ находятся во взаимно однозначном соответствии на множестве $\{\eta<g(1)\})$;

(b) п.н. при событии $\{\eta \geqslant g(1)\}$ распределение сосредоточено на множестве $\{\alpha, \beta\}$ и, более того,

$$
\mathbf{E}(\xi \mid \eta)=1 \text { п.н. на }\{\eta \geqslant g(1)\}
$$

что однозначно определяет условное распределение $\xi$ относительно $\eta$.

Чтобы доказать (2.1), достаточно проверить, что для любого интервала $(a, b) \subset\left[g(1), g^{*}\right]$ выполняется $\mathbf{E} \xi 1_{\{\eta \in(a, b)\}}=\mathbf{P}(\eta \in(a, b))$. Имеем $\{\eta \in(a, b)\}=\{g(R) \in(a, b)\}=\left\{R \in\left(r_{0}, r_{1}\right) \cup\left(r_{2}, r_{3}\right)\right\}$ с $g\left(r_{0}\right)=g\left(r_{3}\right)=a$ и $g\left(r_{1}\right)=g\left(r_{2}\right)=b$, следовательно,

$$
\begin{aligned}
\mathbf{E} \xi 1_{\{\eta \in(a, b)\}} & =\mathbf{E} F^{-1}(R) 1_{\{g(R) \in(a, b)\}}=\left(h\left(r_{1}\right)-h\left(r_{0}\right)\right)+\left(h\left(r_{3}\right)-h\left(r_{2}\right)\right) \\
& =\left(r_{1}-r_{0}\right)+\left(r_{3}-r_{2}\right)=\mathbf{P}\left(R \in\left(r_{0}, r_{1}\right) \cup\left(r_{2}, r_{3}\right)\right) \\
& =\mathbf{P}(\eta \in(a, b))
\end{aligned}
$$

(вспомним определения функций $g$ и $h$ ). Другие утверждения из пунктов (a) и (b) очевидны.

Теперь определим $\tau$ формулой

$$
\tau=\inf \left\{t \in \mathbf{R}_{+}: Y_{t} \notin(\alpha, \beta)\right\} \text {. }
$$

Так как случайные величины $\alpha$ и $\beta \mathscr{F}_{0}$-измеримы, $\tau$ является $\left(\mathscr{F}_{t}\right)$ моментом остановки. Докажем, что условное распределение $Y_{\tau}$ относительно $\eta$ допускает следующую характеризацию:

(А) п.н. при событии $\{\eta<g(1)\}$ распределение $Y_{\tau}$ сосредоточено на одноточечном множестве $\{\xi\}$;

(В) п.н. при событии $\{\eta \geqslant g(1)\}$ распределение $Y_{\tau}$ сосредоточено на множестве $\{\alpha, \beta\}$ и, более того,

$$
\mathbf{E}\left(Y_{\tau} \mid \eta\right)=1 \text { п.н. на }\{\eta \geqslant g(1)\} .
$$

Действительно, если $\mathbf{P}(\eta<g(1))>0$, то $\beta=\infty$ на $\{\eta<g(1)\}$ и, так как $\lim _{t \rightarrow \infty} Y_{t}=0$ п.н., имеем $Y_{\tau}=\alpha=F^{-1}(R)=\xi$ при этом событии (включая случай $\alpha=0$, когда $\tau=\infty$ ). Первое утверждение в пункте (В) понятно. Остается проверить (2.2). Возьмем такое $r \in[0,1]$, что $g(r) \geqslant g(1)$. Так как $V(r)<\infty$ для таких $r$, процесс $\left(Y(\cdot, r)_{t \wedge \tau(\cdot, r)}\right)$ есть ограниченный мартингал на $\mathbf{C}\left(\mathbf{R}_{+}\right)$относительно канонической фильтрации и винеровской меры $\mu_{W}$, т.е. $\int_{\mathbf{C}\left(\mathbf{R}_{+}\right)} Y(x, r)_{\tau(x, r)} \mu_{W}(d x)=1$. 
Это означает, что $\mathbf{E}\left(Y_{\tau} \mid R\right)=1$ п.н. на $\{\eta \geqslant g(1)\}$. Утверждение $(2.2)$ теперь следует из телескопического свойства условного математического ожидания.

Сравнивая (a), (b) и (A), (B), получаем, что условные распределения $\xi$ и $Y_{\tau}$ относительно $\eta$ совпадают. Следовательно, совпадают их безусловные распределения. Лемма доказана.

3 а м е ч а н и е 2.1. (i) На первый взгляд может показаться заманчивым для доказательства леммы 2.1 воспользоваться конструкцией Дуба для вложения в броуновское движение (см. абзац после задачи I в разд. 3). Однако эта идея не сработает, потому что $Y$ невозвратно. А именно, для момента остановки $\tau$, определенного аналогично (3.1), мы, как правило, будем иметь $Y_{\tau} \neq f\left(Y_{1}\right) \sim \xi$.

(ii) Доказательство леммы 2.1 дает альтернативное решение задачи вложения Скорохода для геометрического броуновского движения. Оно напоминает решение Холла [21], хотя качественно отличается от него. И в [21], и в доказательстве выше момент остановки строится как момент достижения одного из двух уровней, $\alpha$ и $\beta$, получаемых с помощью рандомизации. Тем не менее, эти рандомизации очень различаются. Например, если распределение $\xi$ не имеет атомов, то $\beta$ в нашей конструкции всегда является детерминированной функцией от $\alpha$, в то время как в [21] случайный вектор $(\alpha, \beta)$ является «действительно двумерным», т.е. условное распределение $\beta$ при условии $\alpha$ является невырожденным.

(iii) Конструкция из доказательства леммы 2.1 встречалась ранее в статистическом контексте в [9] при доказательстве того, что каждый бинарный эксперимент эквивалентен смеси строго упорядоченных простых бинарных экспериментов. Его аналог для броуновского движения - один из шагов в доказательстве Монро [29]. В нашем случае (геометрическое броуновское движение, математическое ожидание $\xi$ может быть меньше единицы) мы построили аналогичную конструкцию и нашли короткое доказательство с помощью (2.1).

(iv) Другие решения задачи вложения Скорохода для геометрического броуновского движения или, что эквивалентно, для броуновского движения со сносом, были получены в ряде работ, указанных во введении. Еще один способ построить необходимое вложение - это свести задачу к задаче вложения Скорохода для броуновского движения и нецентрированного искомого распределения с помощью замены времени, см. объяснение в разд. 3. Тогда можно использовать решение, предложенное Коксом в [11], или просто запустить броуновское движение, пока оно не достигнет среднего значения распределения, а затем использовать решение в центрированном случае.

(v) Для последующих целей отметим, что уровни $\alpha$ и $\beta$ в доказательстве леммы 2.1 зависят от внешней равномерно распределенной на $[0,1]$ случайной величины $R$ и от распределения $F$, которое мы хо- 
тим вложить: с небольшой натяжкой в обозначениях мы будем писать $\alpha=\alpha(F, R), \beta=\beta(F, R)$ (вспомним рис. 1 и заметим, что функции $h$ и $g$ строятся с помощью $F$ ).

\section{2. Вложение супермартингала с дискретным временем.} Теперь с помощью леммы 2.1 мы вложим неотрицательный супермартингал с дискретным временем в геометрическое броуновское движение. Пусть $\mathbf{N}_{0}:=\mathbf{N} \cup\{0\}$. Мы также будем использовать обозначение $\operatorname{Law}_{\mathbf{P}}(\xi)$ (соответственно $\operatorname{Law}_{\mathbf{P}}(\xi \mid \eta)$ или $\left.\operatorname{Law}_{\mathbf{P}}(\xi \mid \mathscr{G})\right)$ для распределения $\xi$ по мере $\mathbf{P}$ (соответственно для условного распределения $\xi$ относительно $\eta$ или относительно $\mathscr{G}$ по мере $\mathbf{P})$ всякий раз, когда $\xi$ и $\eta-$ случайные величины и $\mathscr{G}$ является $\sigma$-подалгеброй на некотором вероятностном пространстве $(\Omega, \mathscr{F}, \mathbf{P})$.

Лемма 2.2. Пусть $X=\left(X_{n}\right)_{n \in \mathbf{N}_{0}}$ - неотрицательньй супермартингал с $\mathbf{E} X_{0} \leqslant 1$. Рассмотрим вероятностное пространство с фильтраиией $\left(\Omega, \mathscr{F},\left(\mathscr{F}_{t}\right)_{t \geqslant 0}, \mathbf{P}\right)$, где

$\Omega=\mathbf{C}\left(\mathbf{R}_{+}\right) \times[0,1]^{\mathbf{N}_{0}}, \quad \mathscr{F}=\mathscr{B}\left(\mathbf{C}\left(\mathbf{R}_{+}\right)\right) \otimes \mathscr{B}([0,1])^{\otimes \mathbf{N}_{0}}, \quad \mathbf{P}=\mu_{W} \times \mu_{L}^{\mathbf{N}_{0}}$ $u \mathscr{F}_{t}=\bigcap_{\varepsilon>0} \sigma\left(R_{n}, B_{s} ; n \in \mathbf{N}_{0}, s \in[0, t+\varepsilon]\right)$, а случайнье величинь $R_{n}$ и прочесс $B=\left(B_{t}\right)$ на $\Omega$ определеньл следующим образом: если $\omega=\left(x, r_{0}, r_{1}, \ldots\right)$, mо $R_{n}(\omega):=r_{n}, B_{t}(\omega):=x(t)$. B частности, $R_{n}$, $n \in \mathbf{N}_{0}$, - независимье $\mathscr{F}_{0}$-измеримье равномерно распределеннье на $[0,1]$ случайнье величинь, и В является $\left(\mathscr{F}_{t}, \mathbf{P}\right)$-броуновским движением (отметим, что независимость $B u\left(R_{n}\right)$ включается в данное утверждение). Тогда существует неубывающее семейство $\left(\tau_{n}\right)$ таких $\left(\mathscr{F}_{t}\right)$-моментов остановки со значениями в $[0, \infty]$, что прочессы $\left(X_{n}\right)$ $u\left(Y_{\tau_{n}}\right)$ имеют одинаковье распределения, где $Y_{t}=e^{B_{t}-t / 2}, t \geqslant 0$.

Д ок а з а т е л с т в о. Обозначим $F_{0}$ функцию распределения $X_{0}$. Рассмотрим $\left(\mathscr{F}_{t}\right)$-момент остановки

$$
\tau_{0}=\inf \left\{t \in \mathbf{R}_{+}: Y_{t} \notin\left(\alpha\left(F_{0}, R_{0}\right), \beta\left(F_{0}, R_{0}\right)\right)\right\},
$$

где обозначения $\alpha\left(F_{0}, R_{0}\right)$ и $\beta\left(F_{0}, R_{0}\right)$ вводятся в замечании $2.1(\mathrm{v})$. По лемме 2.1 случайные величины $X_{0}$ и $Y_{\tau_{0}}$ одинаково распределены. Заметим также, что семейство $\left(R_{n}\right)_{n \geqslant 1}$ не зависит от $\sigma\left(\tau_{0}, Y_{t} ; t \geqslant 0\right)$ относительно $\mathbf{P}$.

Будем действовать по индукции. Предположение индукции состоит в следующем. Для некоторого $k \in \mathbf{N}_{0}$ мы построили неубывающее семейство $\left(\tau_{n}\right)_{0 \leqslant n \leqslant k}$ таких $\left(\mathscr{F}_{t}\right)$-моментов остановки, что

$$
\operatorname{Law}_{P r}\left(X_{0}, \ldots, X_{k}\right)=\operatorname{Law} \mathbf{P}\left(Y_{\tau_{0}}, \ldots, Y_{\tau_{k}}\right)
$$

(здесь и далее $\operatorname{Pr}$ обозначает вероятностную меру в пространстве, где определена последовательность $\left.\left(X_{n}\right)\right)$ и что

$$
\left(R_{n}\right)_{n \geqslant k+1} \text { не зависит от } \sigma\left(Y_{t}, \tau_{n} ; t \geqslant 0,0 \leqslant n \leqslant k\right) \text { по мере } \mathbf{P} \text {. }
$$


Требуется построить такой $\left(\mathscr{F}_{t}\right)$-момент остановки $\tau_{k+1} \geqslant \tau_{k}$, что выполнены (2.3) и (2.4) с $k+1$ вместо $k$.

В дальнейшем будем работать с такими случайными величинами, как $X_{k+1} / X_{k}$, используя соглашение $0 / 0:=1$ (обратим внимание, что $X_{k+1}=0$ Pr-п.н. на множестве $\left\{X_{k}=0\right\}$, потому что $\left(X_{n}\right)$ является неотрицательным супермартингалом). Заметим, что $\mathbf{E}_{\mathrm{Pr}}\left(X_{k+1} / X_{k} \mid X_{0}, \ldots, X_{k}\right) \leqslant 1 \operatorname{Pr}$-п.н. Идея состоит в том, чтобы вложить $\operatorname{Law}_{\operatorname{Pr}}\left(X_{k+1} / X_{k} \mid X_{0}, \ldots, X_{k}\right)$ в геометрическое броуновское движение $\left(Y_{t+\tau_{k}} / Y_{\tau_{k}}\right)$ с помощью леммы 2.1. Но это требует некоторых дополнительных технических усилий, поскольку $\tau_{k}$ может принимать бесконечное значение.

Рассмотрим регулярную условную функцию распределения $F_{k+1}=$ $\left(F_{k+1}\left(x \mid x_{0}, \ldots, x_{k}\right)\right)_{x, x_{0}, \ldots, x_{k} \in \mathbf{R}_{+}}$случайной величины $X_{k+1} / X_{k}$ относительно $X_{0}, \ldots, X_{k}$. А именно, для каждого $x_{0}, \ldots, x_{k} \in \mathbf{R}_{+}$, $F_{k+1}\left(\cdot \mid x_{0}, \ldots, x_{k}\right)$ - функция распределения вероятностной меры на $\mathbf{R}_{+}$; $F_{k+1}(x \mid \cdot)$ - борелевская функция на $\mathbf{R}_{+}^{k+1}$ для любого $x \in \mathbf{R}_{+}$, и случайная величина $F_{k+1}\left(x \mid X_{0}, \ldots, X_{k}\right)$ есть вариант условной вероятности $\operatorname{Pr}\left(X_{k+1} / X_{k} \leqslant x \mid X_{0}, \ldots, X_{k}\right)$. Определим $\tau_{k+1}$ по формуле

$$
\begin{gathered}
\tau_{k+1}=\tau_{k}+\inf \left\{t \in \mathbf{R}_{+}: \frac{Y_{t+\tau_{k}}}{Y_{\tau_{k}}} \notin\left(\alpha\left(F_{k+1}\left(\cdot \mid Y_{\tau_{0}}, \ldots, Y_{\tau_{k}}\right), R_{k+1}\right),\right.\right. \\
\left.\left.\beta\left(F_{k+1}\left(\cdot \mid Y_{\tau_{0}}, \ldots, Y_{\tau_{k}}\right), R_{k+1}\right)\right)\right\}
\end{gathered}
$$

$\left(\tau_{k+1}:=\infty\right.$ на событии $\left.\left\{\tau_{k}=\infty\right\}\right)$, который является $\left(\mathscr{F}_{t}\right)$-моментом остановки, потому что $R_{k+1} \mathscr{F}_{0}$-измерима и $F_{k+1}\left(\cdot \mid Y_{\tau_{0}}, \ldots, Y_{\tau_{k}}\right)$ известна в момент времени $\tau_{k}$. Отметим, что (2.4) с заменой $k$ на $k+1$ вытекает из формулы для $\tau_{k+1},(2.4)$ и из факта, что $R_{k+1}, R_{k+2}, \ldots$ независимы по мере $\mathbf{P}$. Остается доказать, что

$$
\operatorname{Law}_{P r}\left(X_{0}, \ldots, X_{k+1}\right)=\operatorname{Law}_{\mathbf{P}}\left(Y_{\tau_{0}}, \ldots, Y_{\tau_{k+1}}\right) .
$$

Если $\mathbf{P}\left(\tau_{k}=\infty\right)=1$ (эквивалентно, $\operatorname{Pr}\left(X_{k}=0\right)=1$ ), то $Y_{\tau_{k+1}}=0$ $\mathbf{P}$-п.н. и $X_{k+1}=0$ Pr-п.н., поэтому (2.5) следует из (2.3). Далее мы предполагаем, что $\mathbf{P}\left(\tau_{k}<\infty\right)>0$. Введем вероятностную меру $\mathbf{Q}$ на $(\Omega, \mathscr{F})$ по формуле

$$
\mathbf{Q}(\cdot):=\mathbf{P}\left(\cdot \mid \tau_{k}<\infty\right) .
$$

Будем использовать обозначение $\mathscr{G}:=\sigma\left(Y_{\tau_{0}}, \ldots, Y_{\tau_{k}}\right)$. Легко убедиться, что для любой неотрицательной случайной величины $Z$ имеем

$$
\mathbf{E}_{\mathbf{Q}}(Z \mid \mathscr{G})=\mathbf{E}_{\mathbf{P}}(Z \mid \mathscr{G}) \quad \mathbf{P} \text {-п.н. на }\left\{\tau_{k}<\infty\right\}
$$

(отметим, что $\left\{\tau_{k}<\infty\right\}=\left\{Y_{\tau_{k}}>0\right\} \in \mathscr{G} \mathbf{P}$-п.н.) или, эквивалентно,

$$
\mathbf{E}_{\mathbf{Q}}(Z \mid \mathscr{G})=\mathbf{E}_{\mathbf{P}}(Z \mid \mathscr{G}) \quad \text { Q-п.н. }
$$


На самом деле тождества (2.6) и (2.7) справедливы условно и на $\mathscr{F}_{\tau_{k}}$. Из (2.7) и (2.4) следует, что для $x \in[0,1]$,

$$
\mathbf{Q}\left(R_{k+1} \leqslant x \mid \mathscr{G}\right)=\mathbf{P}\left(R_{k+1} \leqslant x \mid \mathscr{G}\right)=\mathbf{P}\left(R_{k+1} \leqslant x\right)=x, \quad \mathbf{Q} \text {-п.н. }
$$

т.е. по мере $\mathbf{Q}$ условно относительно $\mathscr{G}$ случайная величина $R_{k+1}$ равномерно распределена на $[0,1]$. Пусть теперь $A \in \mathscr{G}$ и $B=\left\{R_{k+1} \leqslant x\right\}$. Тогда из (2.8) следует, что

$$
\mathbf{Q}(A \cap B)=\mathbf{E}_{\mathbf{Q}}\left[1_{A} \mathbf{Q}(B \mid \mathscr{G})\right]=\mathbf{E}_{\mathbf{Q}}\left[1_{A} \mathbf{Q}(B)\right]=\mathbf{Q}(A) \mathbf{Q}(B),
$$

т.е. $\mathscr{G}$ и $R_{k+1}$ независимы по мере $\mathbf{Q}$. Из строго марковского свойства для броуновского движения (например, в форме [35, Chap. III, Theorem 3.1]) можно вывести, что по мере $\mathbf{Q}$ процесс $\left(Y_{t+\tau_{k}} / Y_{\tau_{k}}\right)$ является геометрическим броуновским движением, независимым от $\mathscr{F}_{\tau_{k}}$. Поскольку $\mathscr{G} \subset \mathscr{F}_{\tau_{k}}$ и $R_{k+1}$ является $\mathscr{F}_{\tau_{k}}$-измеримой (даже $\mathscr{F}_{0}$-измеримой), мы получаем, что

$$
\left(Y_{t+\tau_{k}} / Y_{\tau_{k}}\right)_{t \geqslant 0}, \mathscr{G} \text { и } R_{k+1} \text { независимы по мере } \mathbf{Q} \text {. }
$$

Резюмируя, мы имеем:

1) по мере $\mathbf{Q}$ условно относительно $\mathscr{G}$ процесс $\left(Y_{t+\tau_{k}} / Y_{\tau_{k}}\right)$ является геометрическим броуновским движением;

2 ) по мере $\mathbf{Q}$ условно относительно $\mathscr{G}$ случайная величина $R_{k+1}$ равномерно распределена на $[0,1]$;

3) по мере $\mathbf{Q}$ условно относительно $\mathscr{G}$ процесс $\left(Y_{t+\tau_{k}} / Y_{\tau_{k}}\right)$ и случайная величина $R_{k+1}$ независимы (следует из $(2.9)$ ).

Следовательно, по лемме 2.1 для любого $x \in \mathbf{R}_{+} \mathbf{Q}$-п.н. выполняется

$$
\mathbf{Q}\left(\frac{Y_{\tau_{k+1}}}{Y_{\tau_{k}}} \leqslant x \mid \mathscr{G}\right)=F_{k+1}\left(x \mid Y_{\tau_{0}}, \ldots, Y_{\tau_{k}}\right) \text {. }
$$

По (2.6) $\mathbf{P}$-п.н. на $\left\{\tau_{k}<\infty\right\}$ выполняется

$$
\mathbf{P}\left(\frac{Y_{\tau_{k+1}}}{Y_{\tau_{k}}} \leqslant x \mid \mathscr{G}\right)=F_{k+1}\left(x \mid Y_{\tau_{0}}, \ldots, Y_{\tau_{k}}\right) .
$$

Но Р-п.н. на $\left\{\tau_{k}=\infty\right\}\left(\equiv\left\{Y_{\tau_{k}}=0\right\}\right)$ мы имеем $Y_{\tau_{k+1}}=0$, т.е. левая часть $(2.10)$ есть тогда $1_{\{x \geqslant 1\}}$, что совпадает с правой частью соотношения (2.10) на этом событии. Таким образом, (2.10) выполнено $\mathbf{P}$-п.н. на $\Omega$ (а не только на $\left\{\tau_{k}<\infty\right\}$ ). Поскольку $x \in \mathbf{R}_{+}$произвольно, отсюда следует, что $F_{k+1}\left(\cdot \mid Y_{\tau_{0}}, \ldots, Y_{\tau_{k}}\right)$ является вариантом регулярного условного распределения функции $Y_{\tau_{k+1}} / Y_{\tau_{k}}$ (по мере $\mathbf{P}$ ) относительно $Y_{\tau_{0}}, \ldots, Y_{\tau_{k}}$. Комбинируя выше сказанное с $(2.3)$ и определением $F_{k+1}$, получаем (2.5). Шаг индукции доказан.

Таким образом, мы можем построить неубывающее семейство $\left(\tau_{n}\right)$ таких $\left(\mathscr{F}_{t}\right)$-моментов остановки, что процессы с дискретным временем $\left(X_{n}\right)$ и $\left(Y_{\tau_{n}}\right)$ имеют одинаковые конечномерные распределения. Это завершает доказательство леммы. 
2.3. Предельный переход к непрерывному времени. Перейдем к доказательству теоремы 1.1. У нас задан неотрицательный супермартингал с непрерывным временем $\left(X_{s}\right)$ с $\mathbf{E} X_{0} \leqslant 1$. Для каждого $n \in \mathbf{N}$ рассмотрим кусочно-постоянный неотрицательный супермартингал $\left(X_{s}^{n}\right)=\left(X_{2^{-n}\left\lfloor 2^{n} s\right\rfloor}\right)$. Из леммы 2.2 следует существование таких геометрического броуновского движения $\left(Y_{t}^{n}\right)$ и (кусочно-постоянной) замены времени $\left(T_{s}^{n}\right)$ на некотором вероятностном пространстве с фильтрацией $\left(\Omega^{n}, \mathscr{F}^{n},\left(\mathscr{F}_{t}^{n}\right), \mathbf{P}^{n}\right)$, что процессы $\left(X_{s}^{n}\right)$ и $\left(Y_{T_{s}^{n}}^{n}\right)$ имеют одинаковое распределение. Без ограничения общности будем считать, что $\lim _{t \rightarrow \infty} Y_{t}^{n}\left(\omega_{n}\right)=0$ для всех $\omega_{n} \in \Omega^{n}$, и положим $Y_{\infty}^{n}\left(\omega_{n}\right):=0$ для всех $\omega_{n} \in \Omega^{n}$.

Пусть $\mathbf{C}([0, \infty])$ - пространство всех непрерывных функций $z:[0, \infty] \rightarrow \mathbf{R}$ c sup-нормой, и пусть $\mathscr{A}-$ множество всех неубывающих непрерывных справа функций $a:[0, \infty) \rightarrow[0, \infty]$. Определим метрику $\rho$ на $\mathscr{A}$ равенством $\rho\left(a_{1}, a_{2}\right)=d\left(\widehat{a}_{1}, \widehat{a}_{2}\right)$, где $\widehat{a}_{i}=a_{i} /\left(1+a_{i}\right)$ и

$$
d\left(b_{1}, b_{2}\right)=\sum_{k=1}^{\infty} 2^{-k} \int_{0}^{k}\left|b_{1}(t)-b_{2}(t)\right| d t .
$$

Легко проверить, что сходимость в метрике $d$ в пространстве $\widehat{\mathscr{A}}$ всех неубывающих непрерывных справа функций $b:[0, \infty) \rightarrow[0,1]$ эквивалентна поточечной сходимости в каждой точке, в которой предельная функция непрерывна. По теореме Хелли (см., например, [38, гл. III, $\S 2$, теорема 2]) $(\widehat{\mathscr{A}}, d)$ является компактом. Следовательно, $(\mathscr{A}, \rho)-$ компакт.

Положим $\Omega=\mathbf{C}([0, \infty]) \times \mathscr{A}, \mathscr{F}=\mathscr{B}(\mathbf{C}([0, \infty])) \otimes \mathscr{B}(\mathscr{A})$. Пространство $\Omega$ с топологией произведения является полным сепарабельным метрическим пространством. Определим измеримое отображение $f_{n}: \Omega^{n} \rightarrow \Omega:$

$$
f_{n}\left(\omega_{n}\right)=\left(Y^{n}\left(\omega_{n}\right), T^{n}\left(\omega_{n}\right)\right) .
$$

Пусть $\mathbf{Q}^{n}$ есть образ $\mathbf{P}^{n}$ при отображении $f_{n}$. Сначала покажем, что последовательность $\mathbf{Q}_{n}$ вероятностных мер на $(\Omega, \mathscr{F})$ плотна. Достаточно проверить, что проекции $\mathbf{Q}^{n}$ на $\mathbf{C}([0, \infty])$ и на $\mathscr{A}$ плотны. Проекция $\mathbf{Q}^{n}$ на $\mathbf{C}([0, \infty])$ распределена как геометрическое броуновское движение и не зависит от $n$, что влечет плотность проекций на $\mathbf{C}([0, \infty])$. Плотность проекций на $\mathscr{A}$ следует из компактности $\mathscr{A}$. Таким образом, последовательность $\mathbf{Q}^{n}$ плотна. Далее, определим $\mathbf{P}$ как предельную точку этой последовательности. Очевидно, что процесс $\left(Z_{t}\right)_{t \in \mathbf{R}_{+}}$на $\Omega$, определенный как $Z_{t}(z, a)=z(t)$, является (стандартным) геометрическим броуновским движением по мере $\mathbf{P}:$ а именно, процесс $\left(W_{t}\right)_{t \in \mathbf{R}_{+}}$с $W_{t}=\ln Z_{t}+t / 2$, который корректно определен относительно $\mathbf{P}$, является броуновским движением по $\mathbf{P}$. 
Определим процесс $\left(T_{s}\right)_{s \in \mathbf{R}_{+}}$на $\Omega$ как $T_{s}(z, a)=a(s)$ и рассмотрим минимальную непрерывную справа фильтрацию $\left(\mathscr{F}_{t}\right)_{t \in \mathbf{R}_{+}}$на $\Omega$, по отношению к которой $\left(Z_{t}\right)$ согласован и $\left(T_{s}\right)$ - замена времени, т.е.

$$
\mathscr{F}_{t}=\bigcap_{\varepsilon>0} \sigma\left(Z_{u},\left\{T_{s} \leqslant v\right\}: u, v \in[0, t+\varepsilon], s \in \mathbf{R}_{+}\right) .
$$

Остальные шаги доказательства призваны показать следующее:

1) процесс $\left(Z_{T_{s}}\right)$ распределен так же, как и $\left(X_{s}\right)$ при $\mathbf{P}$;

2) процесс $\left(W_{t}\right)-\left(\mathscr{F}_{t}, \mathbf{P}\right)$-броуновское движение, т.е. $W_{t}-W_{s}$ не зависит от $\mathscr{F}_{s}$ по мере $\mathbf{P}$ для любых $s<t, s, t \in \mathbf{R}_{+}$.

Эти два шага доказываются аналогично соответствующим шагам в доказательстве теоремы 2 в [29] с очевидными изменениями.

Можно также дать другое доказательство, используя вариант теоремы (3.2) в [7]. Идея заключается в том, чтобы ввести такую топологию типа устойчивой сходимости на $\Omega$, что второй пункт остается верным при переходе к пределу; однако тогда компактность является нетривиальной задачей.

3. Задача вложения Скорохода, минимальность и вложение процессов.

\section{1. Классическая задача вложения Скорохода и минималь-} ные моменты остановки. Начнем с нескольких замечаний об эволюции постановки задачи вложения для броуновского движения.

Задача I (вложение в броуновское движение, наивная формулировка).

Задано: случайная величина $\xi$ с действительньми значениями.

Найти: такие вероятностное пространство с фильтрацией $\left(\Omega, \mathscr{F},\left(\mathscr{F}_{t}\right)_{t \geqslant 0}, \mathbf{P}\right),\left(\mathscr{F}_{t}, \mathbf{P}\right)$-броуновское движение $B=\left(B_{t}\right)$ и конечньй $\left(\mathscr{F}_{t}\right)$-момент остановки $\tau$, что $B_{\tau} \sim \xi$.

Задача I допускает следующее тривиальное решение. Пусть $f: \mathbf{R} \rightarrow$ $\mathbf{R}$ - такая функция, что $f\left(B_{1}\right) \sim \xi$. Тогда для момента остановки

$$
\tau:=\inf \left\{t \geqslant 1: B_{t}=f\left(B_{1}\right)\right\}
$$

в силу возвратности броуновского движения, имеем $B_{\tau}=f\left(B_{1}\right) \sim \xi$. Это решение приписывается Дубу (см. обсуждение в разд. 2.3 в [30] или в разд. 3.2 в [23]) и предназначено показать, что без дополнительных требований задача тривиальна.

Следовательно, оригинальная формулировка задачи вложения Скорохода содержит некоторые ограничения.

Задача II (задача вложения Скорохода [37] и [36]).

Задано: случайная величина $\xi$ с действительными значениями $c$ $\mathbf{E} \xi=0 u \mathbf{E} \xi^{2}<\infty$. 
Найти: такие вероятностное пространство с фильтрацией $\left(\Omega, \mathscr{F},\left(\mathscr{F}_{t}\right)_{t \geqslant 0}, \mathbf{P}\right),\left(\mathscr{F}_{t}, \mathbf{P}\right)$-броуновское движение $B=\left(B_{t}\right) u\left(\mathscr{F}_{t}\right)$ момент остановки $\tau$ с $\mathbf{E} \tau<\infty$, ито $B_{\tau} \sim \xi$.

Отметим, что момент остановки $\tau$, заданный в (3.1), исключается, потому что для этого момента остановки $\mathbf{E} \tau=\infty$ (если $f$ не тождественная функция, что возможно, только когда $\xi \sim N(0,1))$. Далее, отметим, что если $\mathbf{E} \tau<\infty$, то $\mathbf{E} B_{\tau}=0$ и $\mathbf{E} B_{\tau}^{2}=\mathbf{E} \tau$, следовательно, если имеется требование $\mathbf{E} \tau<\infty$, то в формулировке задачи мы должны предполагать, что $\mathbf{E} \xi=0$ и $\mathbf{E} \xi^{2}<\infty$. Однако эти предположения $(\mathbf{E} \xi=0$ и $\left.\mathbf{E} \xi^{2}<\infty\right)$ являются недостатком постановки задачи II. Например, моменты остановки в оригинальной конструкции Скорохода (см. [37] и [36]) требуют от $\xi$ только наличия конечного среднего, но, как мы только что видели, если мы не предполагаем конечную дисперсию, перестает быть понятным, как выбирать «хорошие» моменты остановки.

Естественный способ выбора «хороших» моментов остановки состоит в требовании их минимальности (вместо требования $\mathbf{E} \tau<\infty$ ) в следующем смысле. Конечный момент остановки $\tau$ называется минимальнылм, если для момента остановки $\sigma$ из того, что $\sigma \leqslant \tau$ и $B_{\sigma} \sim B_{\tau}$, следует, что $\sigma=\tau$ п.н. В контексте задачи вложения Скорохода это требование предложено в [28] (такое понятие минимальности Монро [28] приписывает Дубу) и принимается во многих последующих работах по вложению Скорохода. В частности, для центрированных распределений, минимальность характеризуется в [28] следующим образом.

Теорема 3.1 (Монро [28]). Пусть $\tau$ - такой конечный момент остановки, что $\mathbf{E} B_{\tau}=0$. В этом случае $\tau$ является минимальньм тогда и только тогда, когда прочесс $\left(B_{t \wedge \tau}\right)_{t \geqslant 0}$ равномерно интегрируем.

Эта характеризация оказалась очень полезной. Мы также воспользуемся ею ниже. Далее, отметим, что минимальность для нецентрированных распределений характеризуется в [13], в частности, теорема 3.1 была обобщена на случай $\mathbf{E}\left|B_{\tau}\right|<\infty$. См. также разд. 8 в [30], разд. 3.4 и 4.2 в [23], а также разд. 2.2 в [31] для дальнейшего обсуждения минимальности.

Как итог, работы [28] и [13] инициируют следующую постановку задачи вложения Скорохода.

Задача III (задача вложения Скорохода, Монро [28], Кокс, Хоб$\mathrm{coH}[13])$.

Задано: случайная величина $\xi$ с действительньми значениями.

Найти: такие вероятностное пространство с фильтрацией $\left(\Omega, \mathscr{F},\left(\mathscr{F}_{t}\right)_{t \geqslant 0}, \mathbf{P}\right),\left(\mathscr{F}_{t}, \mathbf{P}\right)$-броуновское движение $B=\left(B_{t}\right)$ и минимальный $\left(\mathscr{F}_{t}\right)$-момент остановки $\tau$, что $B_{\tau} \sim \xi$.

Отметим, что задача III является более общей, чем задача II, в том 
смысле, что каждое решение задачи II есть решение задачи III (если $\sigma \leqslant \tau$ - решения задачи II, то $\mathbf{E} \tau=\mathbf{E} \sigma=\mathbf{E} \xi^{2}<\infty$, т.е. $\sigma=\tau$ п.н., следовательно, $\tau$ - минимальный), но мы больше не предполагаем, что $\mathbf{E} \xi=0$ и $\mathbf{E} \xi^{2}<\infty$.

3.2. Вложение процессов. В этой части мы объясним, почему теорема 1.1 не является следствием работ [28] и [29]. В действительности, непосредственно из результатов Монро получается только следующее (более слабое) утверждение.

Предложение 3.1. Пусть $X=\left(X_{s}\right)_{s \geqslant 0}$ - неотрицательньй мартингал с $\mathbf{E} X_{0}=1$. Тогда существуют такие вероятностное пространство с фильтрацией $\left(\Omega, \mathscr{F}_{1}\left(\mathscr{F}_{t}\right)_{t \geqslant 0}, \mathbf{P}\right),\left(\mathscr{F}_{t}, \mathbf{P}\right)$-броуновское движение $W=\left(W_{t}\right)$ и $\left(\mathscr{F}_{t}\right)$-замена времени $\left(T_{s}\right)$ со значениями в $[0, \infty]$, что прочессьи $\left(X_{s}\right)_{s \geqslant 0} u\left(Z_{T_{s}}\right)_{s \geqslant 0}$ имеют одинаковые распределения, где $Z_{t}=e^{W_{t}-t / 2}, t \geqslant 0$.

Отметим, что неотрицательные супермартингалы $\left(X_{s}\right)$ с $\mathbf{E} X_{0} \leqslant 1$ (см. теорему 1.1 (i)) — важный класс процессов, который, естественно, появляется в различных областях стохастики, таких как финансовая математика или последовательный анализ. Что касается финансовой математики, так называемые супермартингальные дефляторы естественным образом появляются при расширении класса процессов плотности эквивалентных мартингальных мер. В частности, существование строго положительного супермартингального дефлятора - предположение более слабое, чем предположение о существовании эквивалентной (локальной) мартингальной меры, и эквивалентно некоторой форме отсутствия арбитража, см. [24]. Даже если эквивалентная локальная мартингальная мера существует, необходимо использовать супермартингальные дефляторы в задаче максимизации полезности, см. [27]. Что касается последовательного анализа, отметим, например, что если даны две вероятностные меры $\mathbf{P}$ и $\mathbf{Q}$ на пространстве с фильтрацией $\left(\Omega, \mathscr{F},\left(\mathscr{F}_{t}\right)\right)$, то обобщенный процесс плотности $\left(d \mathbf{Q}_{t} / d \mathbf{P}_{t}\right)$ является, вообще говоря, только супермартингалом по $\mathbf{P}$ (а мартингалом - только когда $\mathbf{Q}$ локально абсолютно непрерывна относительно $\mathbf{P}$ ). Таким образом, действительно лучше иметь теорему 1.1, чем просто предложение 3.1.

Отметим также, что недавно в [20] было установлено асимптотическое поведение математического ожидания диффузий, являющихся строгими супермартингалами, с помощью представления их в виде замены времени в геометрическом броуновском движении.

Обращаясь к обсуждению связи с результатами Монро, напомним, что в обеих работах [28] и [29] рассматривается вопрос о том, является ли процесс эквивалентным замене времени в броуновском движении. Разница состоит в том, что, во-первых, в [28], рассматривается только конечная замена времени, состоящая из минимальных моментов 
остановки, в то время как в [29] рассматриваются все конечные замены времени, и, во-вторых, основной результат в [28] не говорит о необходимости условия в отличие от основного результата в [29].

Теорема 3.2 (Монро [28]). Пусть $M=\left(M_{s}\right)_{s \geqslant 0}-$ мартингал $c$ $\mathbf{E} M_{0}=0$. Тогда существуют такие вероятностное пространство с фильтрацией $\left(\Omega, \mathscr{F},\left(\mathscr{F}_{t}\right)_{t \geqslant 0}, \mathbf{P}\right),\left(\mathscr{F}_{t}, \mathbf{P}\right)$-броуновское движение $W=$ $\left(W_{t}\right)$ и конечная $\left(\mathscr{F}_{t}\right)$-замена времени $\left(T_{s}\right)$, что все моменты остановки $T_{s}$ минимальнь и процессь $\left(M_{s}\right) u\left(W_{T_{s}}\right)$ имеют одинаковое распределение.

Теорема 3.3 (Монро [29]). Непрерьвный справа и имеюший предельи слева прочесс $X=\left(X_{s}\right)_{s \geqslant 0}$ является семимартингалом тогда и только тогда, когда существуют такие вероятностное пространство с фильтрацией $\left(\Omega, \mathscr{F},\left(\mathscr{F}_{t}\right)_{t \geqslant 0}, \mathbf{P}\right),\left(\mathscr{F}_{t}, \mathbf{P}\right)$-броуновское движение $W=\left(W_{t}\right)$ и конечная $\left(\mathscr{F}_{t}\right)$-замена времени $\left(T_{s}\right)$, что процессь $\left(X_{s}\right)$ u $\left(W_{T_{s}}\right)$ имеют одинаковое распределение.

Теперь мы знаем, какие процессы могут рассматриваться как замена времени в броуновском движении (теоремы 3.2 и 3.3 ), в то время как было бы интересно понять, какие процессы могут рассматриваться как замена времени в геометрическом броуновском движении $Z=\left(Z_{t}\right)$ из теоремы 1.1 (i). Идея заключается в том, чтобы сначала сделать замену времени в $Z$, чтобы получить броуновское движение, выходящее из единицы (к которому мы можем применить результаты Монро), но мы сможем получить только броуновское движение, остановленное в нуле. Точнее, вводя $A_{t}:=[Z, Z]_{t}=\int_{0}^{t} Z_{r}^{2} d r$, мы определяем замену времени $\tau_{u}:=\inf \left\{r \geqslant 0: A_{r}>u\right\}$ и полагаем

$$
B_{u}^{0}:=Z_{\tau_{u}}, \quad u \geqslant 0 .
$$

Заметим, что $A_{\infty}<\infty$ п.н. и что $\left(\tau_{u}\right)$ строго возрастает по $\left[0, A_{\infty}\right)$ и равна $+\infty$ на $\left[A_{\infty}, \infty\right)$. Таким образом, $B^{0}=\left(B_{u}^{0}\right)_{u \geqslant 0}$ есть броуновское движение, поглощаемое в нуле, с $B_{0}^{0}=1$ (см. [35, Chap. V, $\left.\S 1\right]$ ). Теперь идея заключается в следующем: если мы можем вложить процесс $X$ в $B^{0}$ в том смысле, что $\left(X_{s}\right)$ и $\left(B_{T_{s}}^{0}\right)$ имеют одинаковые распределения для некоторой замены времени $\left(T_{s}\right)$, тогда мы можем вложить $X$ в $Z$ с помощью замены времени $\left(\tau_{T_{s}}\right)$ (см. $(3.2)$ ). В этом месте оказывается очень полезной теорема 3.2, которая приводит к предложению 3.1. А именно, пусть $X$ - неотрицательный мартингал с $\mathbf{E} X_{0}=1$. Применяя теорему $3.2 \mathrm{k}$ мартингалу $X-1$, получим, что $\left(X_{s}\right)$ имеет то же распределение, что и $\left(Y_{T_{s}}\right)$ для некоторого броуновского движения $Y$, начинающегося в единице, и такой замены времени $\left(T_{s}\right)$, что все моменты остановки $T_{s}-$ минимальные. По теореме 3.1 для каждого $s \geqslant 0$ процесс $\left(Y_{u \wedge T_{s}}\right)_{u \geqslant 0}$ равномерно интегрируем. (Условие $\mathbf{E} B_{\tau}=0$ в теореме 3.1 принимает здесь вид $\mathbf{E} Y_{T_{s}}=1$, потому что $Y$ выходит из единицы и $X-$ мартингал.) 
Поскольку $X_{s} \geqslant 0$ п.н., имеем $Y_{T_{s}} \geqslant 0$ п.н., следовательно, равномерно интегрируемый мартингал $\left(Y_{u \wedge T_{s}}\right)_{u \geqslant 0}$ неотрицателен, что влечет

$$
T_{s} \leqslant H_{0}^{Y}:=\inf \left\{u \geqslant 0: Y_{u}=0\right\} \quad \text { п.н. }
$$

Следовательно, $Y_{T_{s}}=Y_{T_{s}}^{0}$ п.н. для всех $s \geqslant 0$, где $Y^{0}:=\left(Y_{u \wedge H_{0}^{Y}}\right)_{u \geqslant 0}$ есть броуновское движение $Y$, остановленное в момент достижения нуля. Таким образом, $X$ может быть вложен в броуновское движение $Y^{0}$, поглощаемое в нуле, т.е. эта идея работает. Далее остаются некоторые технические детали, но уже ясно, что, действительно, предложение 3.1 может быть выведено из результатов Монро, а именно, из теорем 3.2 и 3.1 .

Напротив, такая аргументация перестает быть верной, если попытаться получить теорему 1.1 (i) из теоремы 3.3. Действительно, пусть $X$ - неотрицательный супермартингал с $\mathbf{E} X_{0} \leqslant 1$. Применяя теорему 3.3 к семимартингалу $X-1$, получим, что $\left(X_{s}\right)$ имеет то же распределение, что и $\left(Y_{T_{s}}\right)$ для некоторых броуновского движения $Y$, выходящего из единицы, и замены времени $\left(T_{s}\right)$. Но теперь нет оснований, чтобы требовать от замены времени $T_{s}$ выполнения условия минимальности. Мы должны обосновать, что $T_{s} \leqslant H_{0}^{Y}$ с определенным выше $H_{0}^{Y}$, но раньше необходимое неравенство $T_{s} \leqslant H_{0}^{Y}$ мы получали с помощью минимальности $T_{s}$ вместе со свойством $\mathbf{E} Y_{T_{s}}=1$. В ситуации теоремы 3.3, может получиться, что требуемое неравенство нарушается даже тогда, когда мы рассматриваем неотрицательный супермартингал $X$ с $\mathbf{E} X_{0} \leqslant 1$ (можно легко построить такие примеры, пользуясь возвратностью броуновского движения). Таким образом, нам надо объяснить, что когда $X$ - неотрицательный супермартингал с $\mathbf{E} X_{0} \leqslant 1$, можно не только найти замену времени $\left(T_{s}\right)$ как указано в теореме 3.3 , но и замену времени с дополнительным свойством $T_{s} \leqslant H_{0}^{Y}$. Однако последнее утверждение выходит за рамки теоремы Монро.

Более того, следующее утверждение, которое дополняет теорему 3.2 , является прямым следствием нашей теоремы 1.1.

Теорема 3.4. Пусть $X=\left(X_{s}\right)_{s \geqslant 0}$ - ограниченный снизу супермартингал с $\mathbf{E} X_{0} \leqslant 0$. Тогда есть такие вероятностное пространство с фильтрацией $\left(\Omega, \mathscr{F},\left(\mathscr{F}_{t}\right)_{t \geqslant 0}, \mathbf{P}\right),\left(\mathscr{F}_{t}, \mathbf{P}\right)$-броуновское движение $W=\left(W_{t}\right)$ и конечная $\left(\mathscr{F}_{t}\right)$-замена времени $\left(T_{s}\right)$, что все моменты остановки $T_{s}$ являются минимальныли и прочессьл $\left(X_{s}\right)$ u $\left(W_{T_{s}}\right)$ имеют одинаковое распределение.

Д ок а з а тель с т в о. Пусть $c>0$ и $X_{s} \geqslant-c$ для всех $s \geqslant 0$. По теореме 1.1 , процесс $\left(c^{-1} X_{s}+1\right)_{s \geqslant 0}$ эквивалентен замене времени в геометрическом броуновском движении $\left(Z_{\sigma_{s}}\right)_{s \geqslant 0}$ на вероятностном пространстве с фильтрацией $\left(\widetilde{\Omega}, \widetilde{\mathscr{F}},\left(\widetilde{\mathscr{F}}_{t}\right)_{t \geqslant 0}, \widetilde{\mathbf{P}}\right)$. Положим $A_{t}:=[c Z, c Z]_{t}=$ $c^{2} \int_{0}^{t} Z_{r}^{2} d r$ и $\tau_{u}:=\inf \left\{r \geqslant 0: A_{r}>u\right\}$. Как указано выше, $A_{\infty}<\infty$ п.н. 
и $\left(\tau_{u}\right)$ строго возрастает на $\left[0, A_{\infty}\right)$ и равен $+\infty$ на $\left[A_{\infty}, \infty\right)$. По теореме Дамбиса-Дубинса-Шварца, см. [35, Chap. V, Theorem 1.7], существует такое стандартное броуновское движение $W=\left(W_{t}\right)_{t \geqslant 0}$ на расширении $\left(\Omega, \mathscr{F},\left(\mathscr{F}_{t}\right)_{t \geqslant 0}, \mathbf{P}\right)$ пространства $\left(\widetilde{\Omega}, \widetilde{\mathscr{F}},\left(\widetilde{\mathscr{F}}_{\tau_{t}}\right)_{t \geqslant 0}, \widetilde{\mathbf{P}}\right)$, что для всех $t \geqslant 0$

$$
c+W_{t \wedge A_{\infty}}=c Z_{\tau_{t}} \text { и, следовательно, } c+W_{A_{t}}=c Z_{t} .
$$

Тогда $T_{s}:=A_{\sigma_{s}}$ - замена времени относительно $\left(\widetilde{\mathscr{F}}_{\tau_{s}}\right)$ и, следовательно, относительно $\left(\mathscr{F}_{s}\right)$,

$$
T_{s} \leqslant T_{\infty} \leqslant A_{\infty}=\inf \left\{t \geqslant 0: W_{t}=-c\right\} \quad\left(\equiv H_{-c}^{W}\right)
$$

(в частности, моменты остановки $T_{s}$ конечны), и процесс $\left(X_{s}\right)_{s \geqslant 0}$ эквивалентен $\left(W_{T_{s}}\right)_{s \geqslant 0}$.

Наконец, тот факт, что все $T_{s}, s \geqslant 0$, минимальны, следует (с учетом (3.3)) из теоремы 4.1 или из теоремы 5 в [13].

Вышеизложенное показывает, что теорема 1.1 также может быть получена из теоремы 3.4 (вместо теоремы 3.1 используется теорема 5 в $[13])$.

\section{4. Минимальные моменты остановки для других процес-}

сов. Ранее мы обсуждали минимальные моменты остановки только относительно броуновского движения, но можно аналогично рассмотреть минимальность моментов остановки для любого процесса (ср. с разд. 3.4 в $[23])$.

В этом разделе рассмотрим пространство состояний $(E, \mathscr{E})$, где $E$ есть $[l, r]$ с $-\infty \leqslant l<r \leqslant \infty$ или $\mathbf{R}^{d} \cup\{\infty\}$ и $\mathscr{E}$ - борелевская $\sigma$-алгебра на $E .^{4)}$ То, что пространство состояний содержит бесконечные точки, может оказаться полезным для изучения моментов остановки, которые могут принимать бесконечное значение.

О п р е д е л е н и е 4.1. Пусть $X=\left(X_{t}\right)_{t \geqslant 0}$ - согласованный непрерывный справа и имеющий пределы слева процесс со значениями в $E$ на вероятностном пространстве с фильтрацией $\left(\Omega, \mathscr{F},\left(\mathscr{F}_{t}\right)_{t \geqslant 0}, \mathbf{P}\right)$. Говорят, что $\left(\mathscr{F}_{t}\right)$-момент остановки $\tau$ минимальный для $X$, если для любого $\left(\mathscr{F}_{t}\right)$-момента остановки $\sigma$ из $\sigma \leqslant \tau$ и $X_{\sigma} \sim X_{\tau}$ следует, что $\sigma=\tau$ п.н. Предполагается, что предел $X_{\infty}:=\lim _{t \rightarrow \infty} X_{t}$ существует п.н. на множестве $\{\tau=\infty\}$ всякий раз, когда проверяется минимальность момента остановки $\tau$ с $\mathbf{P}(\tau=\infty)>0$ (так что $X_{\tau}$ и $X_{\sigma}$ корректно определены).

Заметим, что, например, для броуновского движения с ненулевым сносом естественным пространством состояний является $\overline{\mathbf{R}}:=[-\infty, \infty]$. Это позволяет проверить каждый момент остановки на минимальность

4) Что касается топологии на $\mathbf{R}^{d} \cup\{\infty\}$, то в качестве системы окрестностей точки $\infty$ в $\mathbf{R}^{d} \cup\{\infty\}$ берется семейство дополнений компактных множеств в $\mathbf{R}^{d}$. 
и не исключать априори моменты остановки, которые принимают бесконечные значения. В связи с этим отметим также, что для броуновского движения с ненулевым сносом каждый момент остановки является минимальным, как это следует из следующей теоремы (что отличается от случая броуновского движения, ср. с разд. 3$)$.

Теорема 4.1. Пусть $\tau-\left(\mathscr{F}_{t}\right)$-момент остановки и $g: E \rightarrow \overline{\mathbf{R}}-$ измеримая функиия, для которьх выполнено следующее:

(а) остановленный проиесс $g(X)^{\tau}=\left(g\left(X_{t \wedge \tau}\right)\right)_{t \geqslant 0}$ является замкнутылм супермартингалом (т.е. $g(X)^{\tau}$ - супермартингал, ограниченньй снизу равномерно интегрируемым мартингалом),

(b) п.н. $g(X)$ не имеет интервалов постоянства на стохастическом интервале $[0, \tau)$,

(с) п.н. на $\{\tau=\infty\}$ существует $X_{\infty}:=\lim _{t \rightarrow \infty} X_{t}$.

Тогда $\tau$ является минимальньл для $X$.

3 а м е ч а н и е 4.1. Пусть $E=[l, r]$ и функция $g$ строго монотонна. Тогда $\tau$ минимален всегда, когда выполняются только условия (a) и (b) (другими словами, в данном случае условие (c) можно опустить). Действительно, если бы $\left(X_{t}\right)_{t \geqslant 0}$ имел различные предельные точки при $t \rightarrow \infty$ на $\{\tau=\infty\}$, то $\left(g\left(X_{t}\right)\right)_{t \geqslant 0}$ также имел бы различные предельные точки. Но последнее утверждение не имеет место, потому что в силу (а) предел $\lim _{t \rightarrow \infty} g\left(X_{t}\right)$ существует п.н. на $\{\tau=\infty\}\left(g(X)^{\tau}\right.$ сходится п.н. как замкнутый супермартингал).

Д ок а з а т ел ь с т в о т е о р е м ы 4.1. Без ограничения общности далее будем считать, что $g$ - тождественная функция (иначе проведите последующие рассуждения с $g(X)$ вместо $X)$.

Доказательство является комбинацией следующих утверждений.

(1) Для замкнутого супермартингала $Y$ по теореме Дуба о преобразовании свободного выбора для любых моментов остановки $\rho \leqslant \eta$ имеем $Y_{\rho}, Y_{\eta} \in L^{1}$ и $\mathbf{E}\left(Y_{\eta} \mid \mathscr{F}_{\rho}\right) \leqslant Y_{\rho}$ п.н.

(2) Если $\xi_{1} \leqslant \xi_{2}$ - случайные величины из $L^{1}$ с $\mathbf{E} \xi_{1}=\mathbf{E} \xi_{2}$, то $\xi_{1}=\xi_{2}$ п.н.

Предположим, что $\sigma$ - момент остановки, $\sigma \leqslant \tau$ и $X_{\sigma} \sim X_{\tau}$. Тогда, ввиду утверждений $(1)$ и $(2)$, справедливо $\mathbf{E}\left(X_{\tau} \mid \mathscr{F}_{\sigma}\right)=X_{\sigma}$ п.н. Возьмем строго выпуклую функцию $h$ линейного роста, например, $h(x)=$ $\sqrt{1+x^{2}}$. По неравенству Йенсена и утверждению $(2) \mathbf{E}\left(h\left(X_{\tau}\right) \mid \mathscr{F}_{\sigma}\right)=$ $h\left(X_{\sigma}\right)$ п.н., т.е. в неравенстве Йенсена будет равенство со строго выпуклой функцией. Тогда $X_{\tau}=\mathbf{E}\left(X_{\tau} \mid \mathscr{F}_{\sigma}\right)$ п.н., т.е. $X_{\tau}=X_{\sigma}$ п.н.

Пусть $\rho$ - любой момент остановки с $\sigma \leqslant \rho \leqslant \tau$. Тогда

$$
X_{\rho}=\mathbf{E}\left(X_{\tau} \mid \mathscr{F}_{\rho}\right)=\mathbf{E}\left(X_{\sigma} \mid \mathscr{F}_{\rho}\right)=X_{\sigma} \text { п.н., }
$$

где первое равенство следует из утверждений (1) и (2) (надо воспользоваться тем, что $\mathbf{E}\left(X_{\tau} \mid \mathscr{F}_{\sigma}\right) \leqslant \mathbf{E}\left(X_{\rho} \mid \mathscr{F}_{\sigma}\right) \leqslant X_{\sigma}$ п.н.). Поскольку $X$ не имеет интервалов постоянства на $[0, \tau)$, получаем, что $\sigma=\tau$ п.н. 
3 а м е ч а н и е 4.2. Теорема 4.1 может быть слегка обобщена следующим образом. Слово «супермартингал» в утверждении теоремы следует понимать как непрерывный справа и имеющий пределы слева процесс, являющийся супермартингалом в смысле определения (1.1) в [35, Chap. II], и к условиям (a)-(c) должно быть добавлено следующее условие:

(d) $g\left(X_{\tau}\right) \in L^{1}$.

Это немного более общее определение супермартингала (применимое к процессу $Y$ ) отличается от обычного только тем, что требуется выполнение условия $Y_{t}^{-} \in L^{1}, t \geqslant 0$, в то время как $Y_{t}$ может быть неинтегрируемой (и также принимать значение $\infty$ с положительной вероятностью). В результате утверждение становится немного более сильным, чем в теореме 4.1 (в теореме 4.1 условие $(\mathrm{d})$ выполняется автоматически, см. утверждение (1) в доказательстве), но формулировка теоремы 4.1 является более прозрачной в нынешнем виде. То же доказательство применимо с той лишь разницей, что в утверждении (1) у нас есть только $Y_{\rho}^{-}, Y_{\eta}^{-} \in L^{1}$, но, благодаря (d), мы всегда, когда требуется, можем использовать утверждение (2).

В приведенных ниже примерах мы увидим, что теорема 4.1 применима во многих конкретных ситуациях. Нам также понадобится следующая лемма (ее доказательство является очевидным).

Лемма 4.1. Пусть $Y=\left(Y_{t}\right)_{t \geqslant 0}-$ супермартингал. $Y$ - замкнутьии супермартингал тогда и только тогда, когда семейство $\left(Y_{t}^{-}\right)_{t \geqslant 0}$ равномерно интегрируемо.

В примерах 4.1 и 4.2 ниже, $X$ будет одномерной диффузией. Для этого введем некоторые обозначения. Пусть $J=(l, r),-\infty \leqslant l<r \leqslant \infty$, и $E=[l, r]$. Мы рассматриваем однородную во времени диффузию $X$ в $J$, являющуюся решением стохастического дифференциального уравнения

$$
d X_{t}=\mu\left(X_{t}\right) d t+\sigma\left(X_{t}\right) d W_{t}, \quad X_{0}=x_{0} \in J,
$$

на некотором вероятностном пространстве с фильтрацией $(\Omega, \mathscr{F}$, $\left.\left(\mathscr{F}_{t}\right)_{t \geqslant 0}, \mathbf{P}\right)$, где $W-\left(\mathscr{F}_{t}\right)$-броуновское движение. Мы предполагаем, что коэффициенты $\mu$ и $\sigma$ являются борелевскими функциями и удовлетворяют условиям

$$
\begin{aligned}
& \sigma(x) \neq 0 \quad \forall x \in J, \\
& \frac{1}{\sigma^{2}}, \frac{\mu}{\sigma^{2}} \in L_{\mathrm{loc}}^{1}(J),
\end{aligned}
$$

где $L_{\text {loc }}^{1}(J)$ обозначает множество локально интегрируемых на $J$ функций. При выполнении условий (4.2) и (4.3) стохастическое дифференциальное уравнение (4.1) имеет слабое решение, единственное по распределению, которое, возможно, выходит из $J$ (см. [25, разд. 5.5]). Время 
выхода обозначается $\zeta$. То есть п.н. на $\{\zeta=\infty\}$ траектории $X$ не выходят из $J$, в то время как п.н. на $\{\zeta<\infty\}$ либо $\lim _{t}{ }_{\zeta} X_{t}=r$, либо $\lim _{t \nearrow \zeta} X_{t}=l$. Мы задаем поведение $X$ после $\zeta$ на $\{\zeta<\infty\}$, делая $l$ и $r$ поглощающими границами. Таким образом, мы получаем процесс $X=\left(X_{t}\right)_{t \geqslant 0}$ со значениями в $E$. Для некоторого $c \in J$ положим

$$
s(x)=\int_{c}^{x} \exp \left\{-\int_{c}^{y} \frac{2 \mu}{\sigma^{2}}(z) d z\right\} d y, \quad x \in E \quad(\equiv[l, r]),
$$

которая является функцией шкалы процесса $X$ (любая функция шкалы процесса $X$ есть аффинное преобразование $s$ со строго положительным наклоном). Отметим, что на $J$ функция $s$ является строго возрастающей $C^{1}$-функцией со строго положительной абсолютно непрерывной производной, в то время как $s(r)$ (соответственно $s(l))$ может принимать значения $\infty$ (соответственно $-\infty)$. Наконец, напомним, что $s(X)$ является $\left(\mathscr{F}_{t}\right)$-локальным мартингалом (граница, на которой функция шкалы равна бесконечности, не достигается).

П р и м е р 4.1 (одномерная диффузия, невозвратный случай). Предположим, что $s(r) \wedge|s(l)|<\infty$. Тогда $s(X)$ есть локальный мартингал, ограниченный снизу (если $s(l)>-\infty)$ или сверху (если $s(r)<\infty)$, следовательно, является замкнутым супер- или субмартингалом. Из теоремы 4.1 с функцией $g$, равной $s$ или $-s$, следует, что при условии $s(r) \wedge|s(l)|<\infty$

\section{каждый $\left(\mathscr{F}_{t}\right)$-момент остановки $\tau$ такой, что $\tau \leqslant \zeta$, п.н. минимален для $X$.}

(Обратите внимание, что в силу формулы Ито, примененной к $s(X)$, предположение (b) в теореме 4.1 следует из (4.2), в то время как (c) можно не проверять, учитывая замечание 4.1.)

3 а м е ч а н и е 4.3. Пусть $a \neq 0$ и $B-\left(\mathscr{F}_{t}\right)$-броуновское движение на некотором вероятностном пространстве с фильтрацией. Положим $Y_{t}=B_{t}+a t, t \geqslant 0$. Из предыдущего примера следует, что каждый $\left(\mathscr{F}_{t}\right)$-момент остановки минимален для $Y$ (и для геометрического броуновского движения $e^{Y}$ ). В частности, в отличие от броуновского случая, при рассмотрении задачи вложения Скорохода для геометрического броуновского движения $\left(e^{B_{t}-t / 2}\right)$, как мы делали в лемме 2.1 , нет разницы между постановками задачи как задачи I или как задачи III в разд. 3.

П р и м е р 4.2 (одномерная диффузия, возвратный случай). Предположим, что $s(r)=-s(l)=\infty$. Тогда $\zeta=\infty$ п.н. и $\lim \sup _{t \rightarrow \infty} X_{t}=$ $r$ п.н., $\liminf \operatorname{si\infty }_{t \rightarrow \infty} X_{t}=l$ п.н. В частности, в этом примере минимальность определена только для конечных $\left(\mathscr{F}_{t}\right)$-моментов остановки. Предположим сначала, что локальный мартингал $Y:=s(X)$ есть настоящий 
мартингал. Отметим, что $Y$ удовлетворяет стохастическому дифференциальному уравнению

$$
d Y_{t}=\varkappa\left(Y_{t}\right) d W_{t}, \quad Y_{0}=y_{0}:=s\left(x_{0}\right)
$$

где $\varkappa:=\left(s^{\prime} \sigma\right) \circ s^{-1}$ - борелевская функция, удовлетворяющая

$$
\varkappa(x) \neq 0 \quad \forall x \in \mathbf{R}, \quad \varkappa^{-2} \in L_{\mathrm{loc}}^{1}(\mathbf{R}) .
$$

Из [26] следует, что $Y$ является мартингалом тогда и только тогда, когда

$$
\int_{c}^{\infty} \frac{x}{\varkappa^{2}(x)} d x=\infty \text { и } \int_{-\infty}^{c} \frac{|x|}{\varkappa^{2}(x)} d x=\infty
$$

для некоторого $c \in \mathbf{R}$ (условие (4.6) не зависит от выбора $c$ ввиду (4.5)). Теперь теорема 4.1 с функцией $g$, равной $s$ или $-s$, и лемма 4.1 влекут, что при $s(r)=-s(l)=\infty$ и (4.6) любой $\left(\mathscr{F}_{t}\right)$-момент остановки $\tau$, удовлетворяющий условию

$$
\text { либо }\left(s\left(X_{t \wedge \tau}\right)^{-}\right)_{t \geqslant 0}, \text { либо }\left(s\left(X_{t \wedge \tau}\right)^{+}\right)_{t \geqslant 0} \text { равномерно интегрируем, }
$$

является конечным и минимальным для $X$. (Мы также получаем из (4.7), что момент остановки $\tau$ конечен, потому что замкнутый суперили субмартингале $s(X)^{\tau}$ сходится п.н., см. замечание 4.3.) Наконец, если мы более не предполагаем справедливость $(4.6)$, то любой $\left(\mathscr{F}_{t}\right)$ момент остановки $\tau$, удовлетворяющий условию

$$
\text { либо } \mathbf{E} \sup _{t \geqslant 0} s\left(X_{t \wedge \tau}\right)^{-}<\infty, \text { либо } \mathbf{E} \sup _{t \geqslant 0} s\left(X_{t \wedge \tau}\right)^{+}<\infty,
$$

является конечным и минимальным для $X$. (Если выполнено условие (4.8), то $s(X)^{\tau}$ является замкнутым супер- или субмартингалом как локальный мартингал, ограниченный снизу или сверху интегрируемой случайной величиной.)

3 а м е ч а н и е 4.4. Пусть $B-\left(\mathscr{F}_{t}\right)$-броуновское движение на некотором вероятностном пространстве с фильтрацией. Из предыдущего примера следует, что любой $\left(\mathscr{F}_{t}\right)$-момент остановки $\tau$, удовлетворяющий условию

либо $\left(B_{t \wedge \tau}^{-}\right)_{t \geqslant 0}$, либо $\left(B_{t \wedge \tau}^{+}\right)_{t \geqslant 0}$ равномерно интегрируем,

является конечным и минимальным для $B$. Напомним теперь, что по теореме 3 в [13] в предположении $\mathbf{E}\left|B_{\tau}\right|<\infty$ условие (4.9), в действительности, равносильно минимальности $\tau$. (Отметим также, что (4.9) влечет за собой, что $B^{\tau}$ является замкнутым супер- или субмартингалом, следовательно $\mathbf{E}\left|B_{\tau}\right|<\infty$.) Таким образом, для броуновского движения 
достаточное условие (4.9), которое мы получаем в теореме 4.1, оказывается необходимым и достаточным (в предположении $\mathbf{E}\left|B_{\tau}\right|<\infty$ ).

В примерах 4.3 и 4.4 ниже $X$ будет $d$-мерным $\left(\mathscr{F}_{t}\right)$-броуновским движением, выходящим из $x_{0} \in \mathbf{R}^{d}, d \geqslant 2$, на некотором вероятностном пространстве с фильтрацией. Пространством состояний будет $E:=\mathbf{R}^{d} \cup\{\infty\}$. Через $|\cdot|$ обозначим евклидову норму на $\mathbf{R}^{d}$. Хорошо известно, что, если $d>2$, то $\lim _{t \rightarrow \infty} X_{t}=\infty$ п.н., а если $d=2$, то $X$ является возвратным. Напомним также, что для всех $d \geqslant 2$ каждое одноточечное множество в $\mathbf{R}^{d}$ является полярным для $X$.

П р и м е р $4.3\left(\mathrm{BM}^{d}, d>2\right.$, невозвратный процесс). Пусть $d>2$. Возьмем $y \in \mathbf{R}^{d}, y \neq x_{0}$, и $g(x)=|x-y|^{2-d}$, $x \in E$. По формуле Ито $g(X)$ является положительным локальным мартингалом, следовательно, замкнутым супермартингалом. Он имеет строго возрастающую квадратическую вариацию, следовательно, не имеет промежутков постоянства. Теорема 4.1 влечет за собой, что каждый $\left(\mathscr{F}_{t}\right)$-момент остановки $\tau$ является минимальным для $X$.

П р и м е р 4.4 (BM ${ }^{2}$, возвратный процесс). При $d=2$ ввиду возвратности $X$ минимальность определена только для конечных $\left(\mathscr{F}_{t}\right)$ моментов остановки. Возьмем $z \in \mathbf{R}^{2}, z \neq x_{0}$, и положим $g_{z}(x)=$ $\ln |x-z|, x \in E$. Определим процесс $Y_{t}=g_{z}\left(X_{t}\right), t \geqslant 0$. По формуле Ито и характеризационной теореме Леви процесс $Y$ удовлетворяет стохастическому дифференциальному уравнению (4.4) с $\varkappa(x)=e^{-x}$ (и $\left.y_{0}=g_{z}\left(x_{0}\right)\right)$, в частности, $Y$ является локальным мартингалом. Здесь $\varkappa$ удовлетворяет (4.5), но не (4.6), что означает, что $Y$ не является мартингалом. Обозначая $X=\left(X^{1}, X^{2}\right)$ и $z=\left(z^{1}, z^{2}\right)$, имеем $Y=\ln |X-z| \leqslant|X-z| \leqslant\left|X^{1}-z^{1}\right|+\left|X^{2}-z^{2}\right|$, значит, $\mathbf{E} \sup _{s \leqslant t} Y_{s}^{+}<\infty$ для всех $t \in[0, \infty)$. Следовательно, $Y$ является субмартингалом. Теперь теорема $4.1 \mathrm{c}-g_{z}$ в роли $g$ и лемма 4.1 влекут, что любой $\left(\mathscr{F}_{t}\right)$-момент остановки $\tau$, удовлетворяющий условию

$$
\left(\left(\ln \left|X_{t \wedge \tau}-z\right|\right)^{+}\right)_{t \geqslant 0} \text { равномерно интегрируем, }
$$

является конечным и минимальным для $X$ (вновь конечность $\tau$ вытекает из (4.10), потому что замкнутый субмартингал $Y^{\tau}$ сходится п.н.). Более того, теорема 4.1 с $g_{z}$ в роли $g$ влечет, что любой $\left(\mathscr{F}_{t}\right)$-момент остановки $\tau$, удовлетворяющий условию

$$
\mathbf{E} \sup _{t \geqslant 0}\left(\ln \left|X_{t \wedge \tau}-z\right|\right)^{-}<\infty
$$

является конечным и минимальным для $X$. Подводя итог, для двумерного $\left(\mathscr{F}_{t}\right)$-броуновского движения $X$, выходящего из $x_{0} \in \mathbf{R}^{2}$, любой $\left(\mathscr{F}_{t}\right)$-момент остановки $\tau$, удовлетворяющий либо (4.10), либо (4.11) с некоторым $z \in \mathbf{R}^{2}, z \neq x_{0}$, является конечным и минимальным для $X$. 
Гущин А.А., Урусов М. А.

\section{СПИСОК ЛИТЕРАТУРЫ}

1. Acciaio B., Beiglböck M., Penkner F., Schachermayer W., Temme J. A trajectorial interpretation of Doob's martingale inequalities. - Ann. Appl. Probab., 2013, v. 23, № 4, p. 1494-1505.

2. Ané T., Geman H. Order flow, transaction clock, and normality of asset returns. J. Finance, 2000, v. 55, № 5, p. 2259-2284.

3. Ankirchner S., Heyne G., Imkeller P. A BSDE approach to the Skorokhod embedding problem for the Brownian motion with drift. — Stoch. Dyn., 2008, v. 8, № 1, p. 35-46.

4. Ankirchner S., Hobson D. G., Strack P. Finite, integrable and bounded time embeddings for diffusions. - Bernoulli, 2015, v. 21, № 2, p. 1067-1088.

5. Ankirchner S., Strack P. Skorokhod embeddings in bounded time. - Stoch. Dyn., 2011, v. 11, № 2-3, p. 215-226.

6. Barndorff-Nielsen O. E., Shiryaev A. Change of Time and Change of Measure. Hackensack, NJ: World Scientific, 2010, 305 p.

7. Baxter J.R., Chacon R.V. Enlargement of $\sigma$-algebras and compactness of time changes. - Canad. J. Math., 1977, v. 29, № 5, p. 1055-1065.

8. Beiglböck M., Henry-Labordère P., Penkner F. Model-independent bounds for option prices - a mass transport approach. - Finance Stoch., 2013, v. 17, № 3, p. 477-501.

9. Birnbaum A. On the foundations of statistical inference: binary experiments. - Ann. Math. Statist., 1961, v. 32, p. 414-435.

10. Brown H., Hobson D. G., Rogers L. C. G. Robust hedging of barrier options. - Math. Finance 2001, v. 11, № 3, p. 285-314.

11. Cox A. M. G. Extending Chacon-Walsh: minimality and generalised starting distributions. - In: Séminaire de Probabilités XLI, Lecture Notes in Math., 2008, v. 1934, p. $233-264$.

12. Cox A.M.G., Hobson D. G. An optimal Skorokhod embedding for diffusions. Stochastic Process. Appl., 2004, v. 111, № 1, p. 17-39.

13. Cox A. M. G., Hobson D. G. Skorokhod embeddings, minimality and non-centred target distributions. - Probab. Theory Related Fields, 2006, v. 135, № 3, p. 395-414.

14. Cox A. M. G., Hobson D. G., Obtój J. Pathwise inequalities for local time: applications to Skorokhod embeddings and optimal stopping. - Ann. Appl. Probab., 2008, v. 18, № 5, p. 1870-1896.

15. Cox A. M. G., Obtój J. Robust hedging of double touch barrier options. - SIAM J. Financial Math., 2011, v. 2, № 1, p. 141-182.

16. Cox A.M. G., Obtój J. Robust pricing and hedging of double no-touch options. Finance Stoch., 2011, v. 15, № 3, p. 573-605.

17. Cox A. M. G., Wang J. Root's barrier: construction, optimality and applications to variance options. - Ann. Appl. Probab., 2013, v. 23, № 3, p. 859-894.

18. Dolinsky Y., Soner H. M. Martingale optimal transport and robust hedging in continuous time. - Probab. Theory Related Fields, 2014, v. 160, № 1-2, p. 391-427.

19. Grandits P., Falkner N. Embedding in Brownian motion with drift and the AzémaYor construction. - Stochastic Process. Appl., 2000, v. 85, № 2, p. 249-254.

20. Gushchin A, Urusov M., Zervos M. On the submartingale/supermartingale property of diffusions in natural scale. - Труды Математического института им. В. А. Стеклова, 2014, т. 287, с. 129-139.

21. Hall W.J. Embedding submartingales in Wiener processes with drift, with applications to sequential analysis. - J. Appl. Probability, 1969, v. 6, p. 612-632.

22. Hobson D. G. Robust hedging of the lookback option. - Finance Stoch., 1998, v. 2, № 4, p. 329-347.

23. Hobson D. G. The Skorokhod embedding problem and model-independent bounds for option prices. - In: Paris-Princeton Lectures on Mathematical Finance 2010, Lecture Notes in Math., 2011, v. 2003, p. 267-318.

24. Karatzas I., Kardaras $C$. The numéraire portfolio in semimartingale financial models. - Finance Stoch., 2007, v. 11, № 4, p. 447-493.

25. Karatzas I., Shreve S.E. Brownian Motion and Stochastic Calculus. New York: Springer-Verlag, 1991. 
26. Kotani $S$. On a condition that one-dimensional diffusion processes are martingales. In: In memoriam Paul-André Meyer: Séminaire de Probabilités XXXIX, Lecture Notes in Math., 2006, v. 1874, p. 149-156.

27. Kramkov D., Schachermayer $W$. The asymptotic elasticity of utility functions and optimal investment in incomplete markets. - Ann. Appl. Probab., 1999, v. 9, № 3, p. 904-950.

28. Monroe I. On embedding right continuous martingales in Brownian motion. - Ann. Math. Statist., 1972, v. 43, p. 1293-1311.

29. Monroe I. Processes that can be embedded in Brownian motion. - Ann. Probab., 1978 , v. 6 , № 1 , p. $42-56$.

30. Obtój J. The Skorokhod embedding problem and its offspring. - Probab. Surv., 2004, v. 1 , p. 321-390.

31. Obtój J. On Some Aspects of Skorokhod Embedding Problem and its Applications in Mathematical Finance. Lecture Notes, 2014. http://people.maths.ox.ac.uk/ obloj/LectureNotes_v4.pdf

32. Obtój J., Ulmer F. Performance of robust hedges for digital double barrier options. Int. J. Theor. Appl. Finance, 2012, v. 15, № 1, 1250003, 34 p.

33. Pedersen J. L., Peskir G. The Azéma-Yor embedding in non-singular diffusions. Stochastic Process. Appl., 2001, v. 96, № 2, p. 305-312.

34. Peskir G. The Azéma-Yor embedding in Brownian motion with drift. - In: High dimensional probability, II (Seattle, WA, 1999), Progr. Probab., v. 47. Boston, MA: Birkhäuser Boston, 2000, p. 207-221.

35. Revuz D., Yor M. Continuous Martingales and Brownian Motion. Berlin: Springer, 1999, $602 \mathrm{p}$.

36. Skorokhod A. V. Studies in the Theory of Random Processes. Reading, MA: AddisonWesley, 1965, $199 \mathrm{p}$.

37. Скороход А.В. Исследования по теории случайных процессов (стохастические дифференциальные уравнения и предельные теоремы для процессов Маркова). Киев: Киевский гос. ун-т, 1961.

38. Ширяев А.Н. Вероятность. В 2-х кн. М: МЦНМО, 2004, 520 с; 408 с. 